\title{
Análisis de la Relación entre Rentabilidad y Riesgo en la Planeación de las Finanzas Personales
}

\author{
Fernando Villada, Jesús M. López-Lezama y Nicolás Muñoz-Galeano \\ Universidad de Antioquia, Facultad de Ingeniería, Departamento de Ingeniería Eléctrica, Calle 67 No. 53 - \\ 108, Oficina 20-405, Medellín, Colombia (e-mail: fernando.villada@udea.edu.co; jmaria.lopez@udea.edu.co; \\ nicolas.munoz@udea.edu.co)
}

Recibido Mar. 19, 2018; Aceptado May. 24, 2018; Versión final Jun. 18, 2018, Publicado Dic. 2018

\begin{abstract}
Resumen
En este artículo se analiza la relación entre rentabilidad y riesgo asociada a las inversiones en el manejo de las finanzas personales. Se explica la forma de enseñar los conceptos fundamentales mediante su aplicación a través de trabajos semestrales guiados. Para lograrlo, cada estudiante plantea una meta de mediano a largo plazo estableciendo un plan de cinco etapas para alcanzarla: la etapa 1 corresponde a inversión en renta fija, en las etapas 2 y 3 se invierte en renta variable, en la etapa 4 se analiza el concepto de riesgo mediante el manejo adecuado de portafolios de inversión y en la etapa 5 se hace una inversión de libre elección. Los resultados al final del curso muestran que aproximadamente el $70 \%$ de los estudiantes logran llegar a la meta en el plazo establecido, resaltando que lo más importante en todos los casos es la reflexión de las causas del éxito o el fracaso. En general se nota un cambio de actitud hacia el manejo de las finanzas personales. Esto lo muestra una encuesta practicada al comienzo y al final del curso que indica que la proporción de estudiantes con alta probabilidad de lograr la seguridad financiera pasa del $25 \%$ al $76 \%$.
\end{abstract}

Palabras clave: análisis de riesgo; inversión; finanzas personales; trabajo semestral guiado

\section{Analysis of the Relationship between Risk and Investment in Personal Financial Planning}

\begin{abstract}
This paper analyzes the relationship between profitability and risk associated with investments in the management of personal finance. The way to teach the fundamental concepts following its application through guided semester works is explained. To do so each student sets a medium to long-term goal by stablishing a five-stage plan to achieve it: stage 1 corresponds to investment in fixed income, stages 2 and 3 corresponds to variable income, the concept of risk is analyzed in stage 4 through the proper management of investment portfolios, and stage 5 is a free choice investment. The results at the end of the course show that approximately $70 \%$ of students get the goal within the deadline, highlighting that the most important part is the reflection of the causes of success or failure in getting the goal. In general, there is a change in attitude toward the management of personal finance. This is shown by a survey carried out at the beginning and end of the course which indicates that the proportion of students with a high probability of achieving financial security increases from $25 \%$ to $76 \%$.
\end{abstract}

Keywords: risk analysis; investment; personal finance; guided semester work 


\section{INTRODUCCIÓN}

Este artículo tiene por objetivo analizar el riesgo asociado con las inversiones en el contexto del manejo de las finanzas personales y la enseñanza de estos conceptos de forma transversal en los cursos de ingeniería económica o matemáticas financieras. Se explican los fundamentos del valor del dinero en el tiempo, manejo de presupuestos, inversiones en renta fija y renta variable, optimización de portafolios de inversión, y la forma de lograr metas financieras de mediano y largo plazo mediante la aplicación adecuada de estos principios. Las finanzas personales se encargan de estudiar la forma de administrar correctamente el dinero con el fin de garantizar la satisfacción de todas nuestras necesidades actuales y futuras. En ella se cubren todo tipo de decisiones financieras que toman los individuos y los hogares con el fin de conseguir el bienestar material.

El dinero y la salud son los elementos básicos para conseguir este bienestar, por lo cual existen muchas similitudes entre ambos, y al mismo tiempo algunas diferencias. Una de ellas es que, mientras la mayoría de las personas acepta y recurre continuamente a la medicina, son grandes los vacíos en finanzas y, en consecuencia, difícilmente podrá aprovecharlas para resolver sus problemas financieros (Palacios, 2012). Lo irónico del asunto es que los conceptos financieros para invertir bien son simples y fáciles de entender. Estos conceptos parten de estudiar las etapas de la vida (Zicari, 2008), el manejo del presupuesto (Guthrie y Nicholls, 2015), las alternativas de inversión (He y Shi, 2017), hasta la relación entre la rentabilidad y el riesgo asociado a estas alternativas (Aren y Zengin, 2016; Hsiao y Tsai, 2018). Los dos últimos trabajos también analizan la preferencia de las personas con mayor nivel de educación financiera a invertir en activos más riesgosos y sofisticados con el objetivo de obtener mejores rentabilidades, lo cual se convierte en una ventaja para lograr la libertad financiera.

El nivel de rentabilidad obtenida en una inversión según el grado de educación financiera se analiza con mayor profundidad en Bellofatto et al. (2018). El estudio se basa en una muestra de 20.285 inversores, quienes en un periodo de diez años ejecutan más de dos millones de operaciones sobre 13.000 diferentes acciones. Se concluye que inversores con más alta educación financiera son más exitosos en cuanto a rentabilidades obtenidas y concentran sus portafolios en pequeños grupos de acciones, complementando la diversificación a través de fondos de inversión. De forma complementaria, el trabajo de Stolper y Walter (2017) demuestra la existencia de una relación directa entre la educación financiera y el nivel de desarrollo económico de un país, siendo los ciudadanos alemanes los que muestran la mayor cultura financiera del mundo. Sin embargo, Ergun (2018) encuentra un mejor desempeño en los polacos cuando el estudio se acota a estudiantes universitarios, concluyendo que el incremento de los cursos financieros en la etapa de educación universitaria producirá mejores resultados en la formación financiera de las personas.

También se hace necesario resaltar la importancia de relacionar la riqueza con el desarrollo integral del individuo. Nachtigall (2016) hace énfasis en que el objetivo último no es ser rico, sino que se debe aprender a disfrutar lo que se tiene desde el amor, la generosidad y en armonía con las demás áreas de la vida. Mediante la aplicación de 39 pruebas de inteligencia emocional, Nachtigall (2016) trata de desarrollar actitudes, creencias y conductas que permitan establecer una relación equilibrada, productiva y adecuada con el dinero. Concluye que ganar dinero, disfrutarlo, cultivar la salud, tener vínculos afectivos gratificantes y divertirse sanamente son los condimentos de una vida plena, próspera y rica de verdad. En consecuencia, las finanzas personales van más allá de un problema financiero, pues una buena gestión de nuestros recursos debe empezar por el diagnóstico de la condición particular de cada individuo, en aspectos como situación familiar, nivel de ingresos y gastos, deseos de formación, trayectoria profesional, situación laboral, sueños, expectativas futuras y proyecto de vida, entre otros. Una buena educación financiera, como complemento a la educación formal, contribuye a disminuir la inequidad en la distribución de la riqueza que tanto afecta a la humanidad. Como lo afirma Chan (2016), la diferencia entre ricos y pobres no es el dinero sino la educación financiera.

A pesar de la relativa sencillez de los conceptos financieros, los programas a nivel mundial por mejorar la educación financiera de sus ciudadanos muestran unos resultados poco gratificantes. Mientras el $90 \%$ de los estudiantes quieren aprender finanzas, el $80 \%$ de los profesores no se siente apto para enseñar esta asignatura (Kiyosaki, 2012). Kiyosaki reporta la existencia de una crisis educativa por la ausencia de educación financiera en las escuelas, lo cual también lo corrobora Palacios (2012), quien en veintidós años de experiencia en la banca, encontró que pocas personas invierten bien, y eso aplica tanto para directivos como para el resto de inversores individuales. Diversos estudios han mostrado una situación más crítica en países emergentes. Un ejemplo es el trabajo de Garay (2016) quien midió el índice de alfabetismo financiero en Bolivia encontrando que la mayoría de los bolivianos no entienden los conceptos financieros básicos. Dicho trabajo sugiere la necesidad de incorporar el alfabetismo financiero en los currículos escolares, incentivando la educación financiera, a fin de reducir las brechas en el manejo de las finanzas personales de los individuos. 
En el caso colombiano, se evidencia la falta de instrumentos válidos y confiables para diagnosticar los contenidos de los programas de educación económica y financiera, razón por la cual el trabajo de Vargas y Avendaño (2014) se diseñó un instrumento que permita evaluar las competencias básicas en educación financiera en estudiantes mayores de 15 años que cursaban primero y segundo semestre de formación universitaria. Como resultado se aportó información importante para la toma de decisiones en cuanto a la mejora de estos programas, pero quedaron inquietudes respecto al diagnóstico en otras edades o etapas de formación. Dadas las falencias mostradas, en este artículo se propone incluir la formación en finanzas personales dentro de los cursos de ingeniería económica o matemáticas financieras. Las estrategias más factibles de ser utilizadas son los proyectos de aula o los trabajos semestrales guiados que tal como lo describen Torres et al. (2016) y Valderrama y Valderrama (2014), permiten generar nuevas destrezas para enfrentar situaciones de la vida cotidiana a partir de los conocimientos teóricos adquiridos.

Este artículo propone el uso de trabajos semestrales guiados para enseñar finanzas personales buscando contribuir a la formación integral del estudiante. Se parte del análisis de casos de la vida cotidiana donde el escenario es el ahorro y el manejo adecuado del dinero para luego enfatizar en la importancia de la educación financiera. Seguidamente, se propone que cada estudiante piense en un sueño de mediano o largo plazo (5 o más años), investigue el valor actual y futuro de dicho sueño para luego generar la estrategia para conseguirlo. El proyecto termina con una evaluación grupal de los resultados reflexionando sobre el éxito o fracaso en la consecución del sueño y los motivos que llevaron a estos resultados. En la mayoría de los casos, los estudiantes toman conciencia de la importancia de establecer metas para el manejo adecuado de las finanzas personales, las estrategias para cada objetivo y muy especialmente la relación entre el riesgo y objetivos de inversión.

\section{JUSTIFICACIÓN Y CONTEXTO}

El principal objetivo del estudio de las finanzas personales es entender lo que sucede en nuestro entorno económico, la forma en que nos afecta y como aprovechar estos conceptos para el beneficio propio y el logro de nuestras metas. A continuación, se muestra la forma de enseñar finanzas personales en el contexto de un curso de ingeniería económica o matemáticas financieras mediante el uso de trabajos semestrales guiados. Tal como lo afirma Valderrama y Valderrama (2014), éstos facilitan la comprensión del mundo y la relación con él mediante el uso y la integración de conocimientos, fortaleciendo los procesos cognitivos y formando en los estudiantes una cultura investigativa.

Se parte de lecturas seleccionadas para contextualizar a los estudiantes con los elementos fundamentales de las finanzas personales, la seguridad financiera, la importancia de realizar un presupuesto objetivo, las etapas de la vida financiera de la persona, las metas personales y en especial, la relación entre las alternativas de inversión para alcanzar estas metas y el nivel de riesgo asociado. Adicionalmente, se realiza un diagnóstico preliminar sobre el manejo de las finanzas personales de cada estudiante mediante una encuesta, la cual se vuelve a aplicar al final del curso con el fin de evaluar el progreso del grupo en educación financiera.

\section{Análisis de lecturas seleccionadas sobre temas de relevancia en finanzas personales}

Se distribuyen artículos actualizados sobre temas relevantes a las finanzas personales. Se parte de lecturas referentes a recomendaciones para vivir con un salario mínimo, el costo y la importancia de la educación, la situación pensional, la rentabilidad y riesgo asociado a las inversiones, e historias de personas exitosas, entre otros. El análisis por grupos de dos o tres personas permite concientizar a los estudiantes de la importancia de las finanzas personales, constituyéndose en un asunto de relevancia para todas las personas de todas las edades.

A partir del análisis de artículos o libros relacionados con la forma de vivir con un salario mínimo, alternativas de inversión, fracasos por asumir grandes riesgos y ejemplos de personas exitosas se concluye que se puede ahorrar independiente del salario recibido. Una buena planificación financiera, perseverancia en el ahorro, conocimiento adecuado de las alternativas de inversión y forma de aprovecharlas y la conciencia de los riesgos asociados a las inversiones permite lograr las metas financieras de corto, mediano y largo plazo.

\section{MARCO TEÓRICO}

Finanzas personales pueden ser definidas como el estudio de la forma en que las personas y los hogares manejan los ingresos necesarios para soportar sus necesidades actuales y el bienestar en el futuro de todos los miembros del hogar. Ellas requieren del manejo de conceptos básicos tales como el valor del dinero en el tiempo, la seguridad financiera, elaboración de presupuestos, el ciclo de vida de las personas, las metas personales, las alternativas de inversión y la relación entre la rentabilidad y riesgo asociado a cada una de estas alternativas (Altfest, 2017). 


\section{La seguridad financiera}

El análisis de las lecturas seleccionadas permite concluir la importancia de la seguridad financiera, es decir, la habilidad de las personas o familias para manejar las necesidades económicas venideras (corto plazo) y prepararse para el futuro (mediano y largo plazo). De igual forma, es necesario prepararse para eventos no deseados como puede ser una enfermedad, la pérdida del empleo, un accidente. Se requiere adquirir el conocimiento necesario y embarcarse en acciones prudentes en cuatro aspectos principales: manejar los ingresos, controlar las deudas, gestionar el riesgo y acumulación de riqueza. Para lograrlo se empieza con cambiar la actitud acerca del dinero y la forma de manejarlo, establecer metas financieras alcanzables de corto, mediano y largo plazo, establecer un plan de ahorro e inversión, controlar los gastos, revisar periódicamente las metas, asegurarse contra imprevistos y aprender a detectar señales de alerta.

\section{Elaboración del presupuesto de gastos personal o familiar}

El presupuesto consiste en listar los ingresos y los egresos en un periodo de tiempo, permitiendo tener el control de los gastos, identificar problemas potenciales antes de su ocurrencia y que se vuelvan inmanejables, planear adecuadamente el futuro y de esta forma poder lograr metas personales de corto, mediano y largo plazo. Todo esto se logra con un presupuesto de gastos inferiores a los ingresos y un manejo racional de las deudas, lo que permite vivir con mayor flexibilidad y tranquilidad. A pesar de que los ahorros no son realmente un gasto, estos juegan un papel central en la elaboración del presupuesto (Guthrie y Nicholls, 2015). Un aspecto clave es lograr tener el control de los gastos, los cuales se pueden dividir en obligatorios y no necesarios. Los primeros corresponden a los necesarios para poder llevar una vida normal entre los que están el pago de la vivienda (hipoteca o arriendo), alimentación, servicios públicos, transporte, seguridad social y material de aseo. Los segundos están relacionados con los caprichos y antojos, los cuales, aunque no debemos evitarlos completamente, si es necesario tener control sobre ellos.

\section{Ciclo de vida de los inversionistas}

El principal problema de las finanzas personales es distribuir adecuadamente el ingreso obtenido en su vida productiva para gastarlo el resto de su vida, donde la incertidumbre por el extenso periodo de tiempo y aspectos particulares de cada persona son factores determinantes. El ciclo de vida humano se puede dividir en 3 etapas (Zicari, 2008): (i) preparación, donde la persona estudia y se capacita para adquirir lo que se puede llamar capital humano; (ii) acumulación, que corresponde a la etapa productiva del individuo donde el capital humano se va convirtiendo en capital financiero; (iii) desacumulación, donde la persona ya no cuenta con un capital humano significativo, pero sí dispone de un capital financiero relevante para consumirlo gradualmente en su etapa de retiro.

El ciclo de vida de los inversionistas empieza con el periodo productivo, donde se debe establecer un plan de inversión partiendo del ahorro fijo mensual como rubro importante dentro del presupuesto. La clave del éxito es la cantidad ahorrada y la escogencia acertada de las inversiones. Entre las alternativas de inversión se tiene renta fija, renta variable, divisas, propiedad raíz, negocio propio u otras más exóticas que dependerán del grado de tolerancia al riesgo de la persona y la etapa del ciclo de vida donde se encuentre.

\section{Metas personales}

Diversos trabajos han mostrado la relación directa entre las metas personales y la preocupación del individuo por alcanzarlas. Chang et al. (2017) estudiaron la frecuencia con que las personas monitorean el progreso en sus finanzas personales, encontrando que esta frecuencia es mayor dependiendo de la importancia de la meta para el individuo y del desempeño de sus ahorros e inversiones. A medida que se tiene un pobre desempeño, la preocupación es mayor induciéndolo a tomar medidas correctivas tal como lo explica la teoría de control.

Las metas personales son el punto de partida para lograr con éxito el manejo de las finanzas personales. Son estas metas la razón de ser o la motivación para alcanzar los objetivos de la vida. Se pueden tener objetivos de corto, mediano y largo plazo que pueden ir desde ahorrar para la cuota inicial de una vivienda o asegurar la educación de los hijos hasta mejorar los ingresos a la edad de retiro o pensionarse anticipadamente. Estos objetivos se consiguen a partir de planes de ahorro e inversión adecuados y asumiendo los riesgos asociados a cada alternativa de inversión y el plazo establecido.

\section{METODOLOGÍA}

El estudio se realizó sobre una muestra de 97 estudiantes conformada por la totalidad de alumnos de un curso de ingeniería económica por cuatro semestres consecutivos durante los años 2016 y 2017. Se utilizó la 
estrategia de trabajos semestrales guiados con el fin de que los estudiantes asimilen los conceptos básicos de las finanzas personales y lo más importante, el riesgo asociado a cada alternativa de inversión en el desarrollo de las etapas para lograr las metas personales.

\section{Intervención}

Durante el proceso de conseguir su meta financiera, los estudiantes aplican la estrategia de trabajos semestrales guiados. Para lograrlo se definieron cinco etapas: inversión en renta fija, inversión en renta variable (dos etapas), inversión en portafolios y una última etapa de inversión de libre elección. Esta estrategia, explicada con mayor detalle en la sección de resultados, es verificada con informes de avance en cada etapa y se convierte en un instrumento valioso para complementar la enseñanza de las finanzas personales de forma práctica y amena, permitiendo una mayor aprehensión del conocimiento.

\section{Instrumento de investigación}

La evaluación de la estrategia pedagógica se realizó mediante una encuesta aplicada al comienzo y al final del curso, la cual fue adaptada de un grupo de cuestionarios propuestos por Nachtigall (2016) y Samper et al. (2010). Este instrumento mostrado en el anexo, consta de 25 preguntas cerradas que se responden marcando verdadero "V" o falso "F", donde el número de "V" marcados es directamente proporcional a la probabilidad de lograr la seguridad financiera.

Los resultados de las encuestas fueron procesados y analizados utilizando Microsoft Excel para Windows 2013, siguiendo el criterio propuesto por Samper et al. (2010) para medir la posibilidad de lograr la seguridad financiera. Un número de "V" marcados por encuesta entre 1 y 7 , es interpretado con una baja probabilidad de lograr la seguridad financiera; un resultado entre 8 y 12, significa mediana probabilidad; ésta se hace alta para un número de "V" entre 13 y 18; y muy alta para una cantidad mayor a 18.

\section{RESULTADOS}

Los resultados del trabajo semestral guiado son mostrados como informes de avance por cada uno de los estudiantes. De esta forma, se tiene un informe preliminar y análisis del desempeño de las inversiones en cada una de las etapas requeridas para cumplir la meta financiera.

\section{Informe preliminar}

Después del análisis de los elementos básicos de las finanzas personales, cada estudiante debe establecer una meta personal de mediano o largo plazo (mayor a cinco años), es decir, aquello que más lo motiva a actuar una vez empiece su ciclo laboral o etapa productiva. Adicionalmente debe consultar el valor actual de ese sueño en el mercado con el fin de generar la estrategia adecuada para conseguirlo.

En el primer informe, cada estudiante debe especificar su sueño o meta financiera a desarrollar en el trabajo semestral guiado, el plazo, el valor actual de la misma y el porcentaje del ingreso a ahorrar para conseguir su objetivo. También se solicita que determine el ahorro acumulado que se requiere al final del plazo establecido mediante la aplicación de la ecuación 1. Donde, el valor futuro equivalente $(F)$ a una cantidad monetaria de hoy $(P)$ con una tasa de interés por periodo $(I)$ para $n$ periodos, se calcula como:

$$
F=P(1+i)^{n}
$$

En este primer informe, el estudiante debe determinar el valor actual de su meta mediante la investigación del mercado y consultar la tasa de interés a aplicar, que en este caso sería la inflación esperada en el plazo establecido para la meta.

\section{Análisis etapa 1: Inversión en renta fija}

En el informe preliminar se definió el sueño o meta personal de cada estudiante, el plazo para alcanzarlo, la cantidad a ahorrar acorde al presupuesto individual y los valores actuales y futuros del sueño. Sigue entonces la planeación financiera que permita lograr la meta en el plazo establecido, en la cual, partiendo del ahorro determinado, se deben seleccionar las alternativas de inversión que permitan acumular los recursos suficientes para cumplir el sueño. Se propone que cada estudiante establezca un plan de cinco etapas, donde la primera corresponde a inversión en renta fija, las etapas 2 y 3 se invierta en renta variable (acciones 0 índices), en la etapa 4 se analice el concepto de riesgo mediante el manejo adecuado de portafolios de inversión y la etapa 5 sea de libre elección. 
En el contexto del trabajo de semestre guiado se asume que cada estudiante empieza su vida laboral con un ingreso promedio de tres salarios mínimos colombianos, correspondiente aproximadamente al salario promedio de un ingeniero recién egresado en Colombia. El ahorro mensual en la primera etapa se destinará a inversión en renta fija por un periodo de un año, que constituirá el punto de partida para reunir el capital suficiente que permita entrar a la segunda etapa de inversión activa o especulativa. Se introduce el concepto de mercado de renta fija, medio por el cual las empresas y el Estado financian sus necesidades con recursos ajenos, pagando a su tenedor una tasa de rentabilidad preestablecida para su plazo de vigencia. Los inversores encuentran diversas alternativas de inversión en este mercado, las cuales van desde bajos montos y corto plazo como cuentas de ahorro a la vista, planes de ahorro programado y los certificados de depósito a término (CDT); hasta inversiones mayores de mediano y largo plazo como son los bonos.

A pesar de que las rentabilidades en este tipo de inversión son inferiores a las de otros productos financieros, se tiene la ventaja de un menor riesgo dado que la tasa de interés es garantizada y acorde al respaldo de la entidad que ofrece el producto; adicionalmente, los planes de ahorro programado tienen otros aspectos positivos como la no exigencia de saldos mínimos, la exención del pago de cobros administrativos, beneficios tributarios y descuento directo de la nómina. Para determinar el valor futuro de un ahorro periódico en este tipo de inversiones se utiliza la ecuación 2, donde $A$ es el ahorro constante entre los periodos 1 y $n, i$ es la tasa interés por periodo y $F$ es la cantidad acumulada en el futuro.

$$
F=A \frac{(1+i)^{n}-1}{i}
$$

El trabajo del estudiante en esta etapa consiste en consultar las rentabilidades ofrecidas por el mercado en al menos cinco alternativas de inversión en renta fija y determinar la más adecuada para su caso dependiendo del ahorro mensual, el riesgo de cada una y el plazo establecido. En el informe se debe determinar el ahorro acumulado en esta primera etapa utilizando la ecuación 2.

\section{Análisis etapas 2 y 3: Inversión en renta variable}

Las siguientes etapas del trabajo consisten en invertir el capital acumulado en alternativas de mayor rentabilidad, aunque asumiendo mayor riesgo, como son: acciones, índices bursátiles y carteras de inversión. En las etapas 2 y 3 se invierte de forma temporal en activos o bienes con tendencia a subir de precio, permitiendo el apalancamiento para incrementar rentabilidad, pero a la vez incrementando el riesgo. Se introduce el tema de mercado de renta variable representado principalmente por las acciones de empresas que cotizan en una bolsa de valores, el cual como su nombre lo indica, no garantiza un rendimiento; sin embargo, aplicando una buena estrategia de negociación, ese rendimiento puede resultar superior al obtenido con renta fija. Se explican las dos estrategias de negociación más utilizadas a saber: análisis técnico y análisis fundamental, las cuales, a pesar de partir de principios simples, requieren bastante experticia para ser aplicadas.

Por esta razón, se hace énfasis en las ventajas de la inversión pasiva, la cual consiste en intentar mantener en todo momento un portafolio equivalente al representado por la totalidad del mercado financiero global. En el trabajo de He y Shi (2017) se demuestra que la inversión pasiva a través de un índice puede superar la rentabilidad obtenida mediante la inversión activa en acciones, especialmente para inversores menos sofisticados. Esto se explica por los mayores costos en comisiones que acarrea la inversión activa dado el cambio frecuente en los activos negociados. Por simplicidad se propone que la inversión en estas dos etapas se realice en el índice SP500 que se calcula a partir de las 500 empresas con mayor capitalización bursátil de la bolsa de valores de Nueva York. La rentabilidad obtenida a través de la inversión en este índice se puede determinar a partir de su histograma elaborado con los datos de precios de cierre desde su creación (Damodaran, 2018). La figura 1 muestra claramente que este histograma tiene un sesgo hacia la derecha con rentabilidades positivas, una mediana de aproximadamente un $14 \%$ y una mayor probabilidad de obtener una rentabilidad del $20 \%$. La rentabilidad obtenida en las etapas 2 y 3 se simula introduciendo en una bolsa 100 balotas que siguen la distribución de rentabilidad del histograma mostrado en la figura 1. Cada estudiante en cada una de estas etapas saca aleatoriamente una balota que determinará la rentabilidad obtenida en el periodo respectivo, aproximándose de esta forma a la situación real de la inversión pasiva en el índice SP500.

\section{Etapa 4: Análisis del riesgo y la rentabilidad de la inversión}

Desde el punto de vista de las finanzas personales, el riesgo se puede definir como la posibilidad de que la rentabilidad de una inversión no se dé como está prevista y hay que tener en cuenta que los mercados premian el riesgo asumido con mayor rentabilidad; de ahí que a pesar que siempre se busquen buenas rentabilidades, se debe desconfiar cuando éstas son muy altas ya que el riesgo puede ser hasta de perder la totalidad del capital invertido (Villada et al., 2017). 


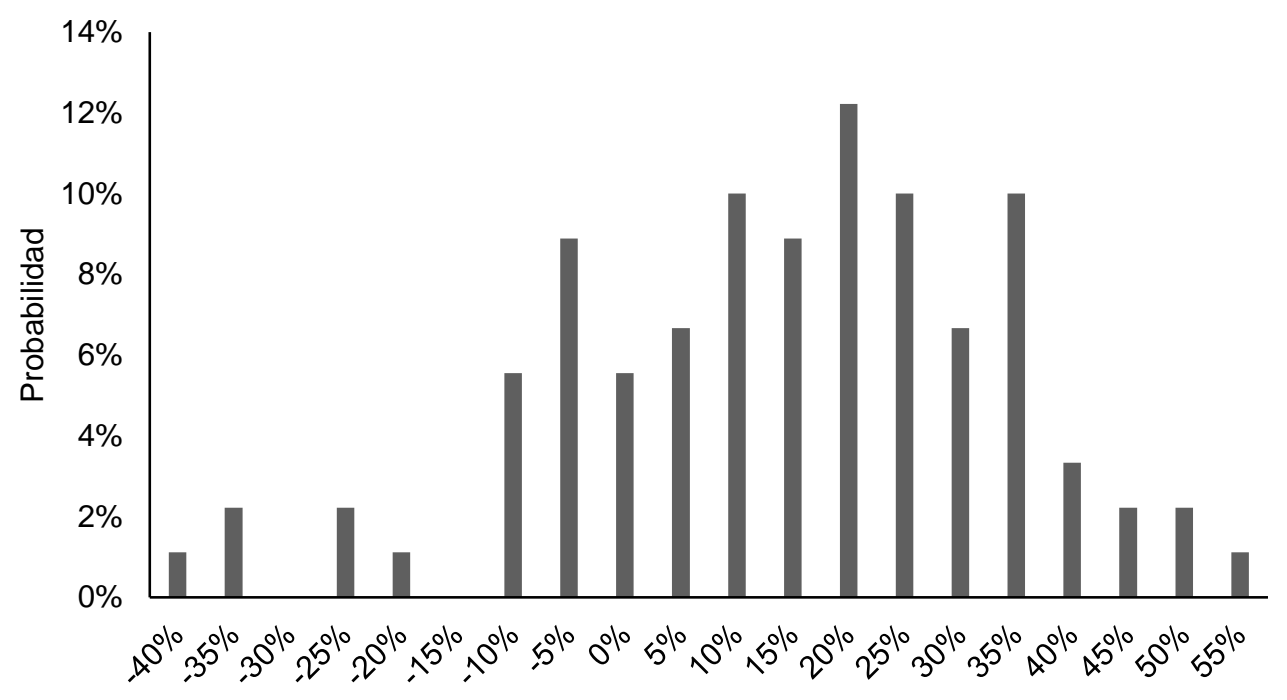

Rentabilidad

Fig1: Histograma de rentabilidad del índice SP500 desde el año 1928.

Diversos autores han investigado la relación entre la preferencia al riesgo y las decisiones de inversión de los individuos, encontrando que estas decisiones no son afectadas por sus rasgos de personalidad. Lo que si se resalta es la forma como la aversión al riesgo y el nivel de educación financiera afectan las decisiones de inversión. Aren y Zengin (2016) encontraron que las personas con alto grado de educación financiera, prefiere invertir en acciones y portafolios al igual que los individuos con alta tolerancia al riesgo, a diferencia de los demás que prefieren depósitos bancarios. El efecto de la educación financiera en inversiones más sofisticadas y de mayor riesgo es analizado por Hsiao y Tsai (2018). En su trabajo tomaron los resultados de una encuesta nacional realizada por la comisión de supervisión financiera de Taiwan para investigar el grado de participación en el mercado de derivados como función de la educación financiera de los individuos. Como resultado se encontró que las personas con alto grado de educación financiera tienen mayor inclinación a comprar productos derivados, lo cual puede ser de gran utilidad para los legisladores y reguladores a nivel mundial para promocionar y entender productos cada vez más sofisticados y el riesgo asociado.

La etapa 4 se considera la más significativa del trabajo del semestre, pues el estudiante debe analizar el riesgo asociado a cada uno de los activos de inversión considerados hasta el momento y lo más importante, la rentabilidad asociada al riesgo asumido. En esta etapa también conocida como de inversión pasiva, se busca invertir de forma permanente en activos que generen la rentabilidad adecuada en el largo plazo para el nivel de riesgo asumido por el inversionista. El mejor ejemplo es la inversión en portafolios que representen uno o varios mercados financieros, buscando disminuir el riesgo a través de la diversificación.

Se presenta el concepto de carteras de inversión (Marcowitz, 1952), la forma de optimizar la distribución de activos en una cartera y se tiene la oportunidad de investigar el uso de diferentes paquetes de software de optimización. De acuerdo con la formulación del modelo de Marcowitz, la tasa esperada de rentabilidad de una cartera para un periodo de tiempo se puede calcular como la suma ponderada de los montos invertidos en los activos que la componen según la ecuación 3.

$E\left(r_{p}\right)=\sum_{i=1}^{N} w_{i} r_{i}$

Donde $r_{i}$ es el rendimiento del activo $i$, y $w_{i}$ es el peso ponderado que tiene el activo $i$ en el portafolio, de manea que $E\left(r_{p}\right)$, es el rendimiendo del portafolio compuesto por $N$ activos.

El riesgo del portafolio se puede calcular por medio de su desviación estándar de acuerdo con la ecuación 4.

$\sigma_{p}=\sqrt{\sum_{i=1}^{N} \sum_{k=1}^{N} w_{i} w_{k} \sigma_{i k}}$

Donde $\sigma_{i k}$ es la covarianza de los rendimientos de los activos, cuando $i \neq k$. Si $i=k$ esta pasa a ser la varianza propia del activo. 
El modelo de Marcowitz puede cumplir dos objetivos dependiendo de las preferencias del inversor: maximizar su rendimiento sujeto a un valor límite del riesgo; o minimizar el riesgo sujeto a un rendimiento esperado. Asumiendo por ejemplo que el inversor se inclina por la primera alternativa, el modelo debe resolver el siguiente problema de optimización:

$$
\operatorname{Max}\left(E\left(r_{p}\right)\right)
$$

Sujeto a restricciones dadas por el máximo riesgo tolerado en el portafolio $\sigma_{p m}$, que los pesos $w_{i}$ deben ser positivos y la sumatoria de los pesos no puede superar el $100 \%$ de los recursos disponibles para invertir. Estas restricciones son representadas en las ecuaciones 6,7 y 8.

$\sigma_{p} \leq \sigma_{p m}$

$\sum_{i=1}^{N} w_{i}=1$

$w_{i} \geq 0, \forall i$

El trabajo a desarrollar en esta etapa consiste en seleccionar una lista de activos de inversión nacionales e internacionales de renta fija y renta variable. A partir del análisis de los rendimientos de los últimos cinco años, se debe calcular la utilidad esperada de cada activo y el riesgo asociado mediante la desviación estándar, para finalmente determinar la composición óptima del portafolio resolviendo el modelo de Marcowitz utilizando un programa de optimización de libre elección por el estudiante.

Se toma por ejemplo el caso de un estudiante que eligió distribuir el capital acumulado hasta el momento en una cartera compuesta por los índices de las bolsas de valores de Nueva York (SP500), Alemania (DAX), Tokio (NIKKEI), México (IPC), Chile (IPSA), Colombia (COLCAP), y una porción en renta fija mediante bonos del tesoro de los Estados Unidos. Al resolver el problema de optimización usando las ecuaciones 5 a 8 , con un nivel de riesgo restringido a un máximo del $4 \%$, se obtuvo una cartera óptima con rentabilidad efectiva anual del $14.88 \%$ y con la distribución de la inversión por activos mostrada en la figura 2.

\section{Etapa 5: Libre elección de alternativa de inversión}

En esta última etapa del trabajo, el estudiante ha experimentado diversas alternativas de inversión y está próximo a cumplir con el plazo establecido para lograr su objetivo, por esta razón su elección aquí es libre dependiendo de su experiencia en las etapas anteriores, la viabilidad de cumplir la meta y el riesgo que esté dispuesto a asumir en esta fase final. Por ejemplo, una persona con baja tolerancia al riesgo o que esté muy próxima o haya cumplido su meta, escogería invertir todos sus recursos en renta fija; mientras que los demás podrían escoger invertir en acciones o portafolios.

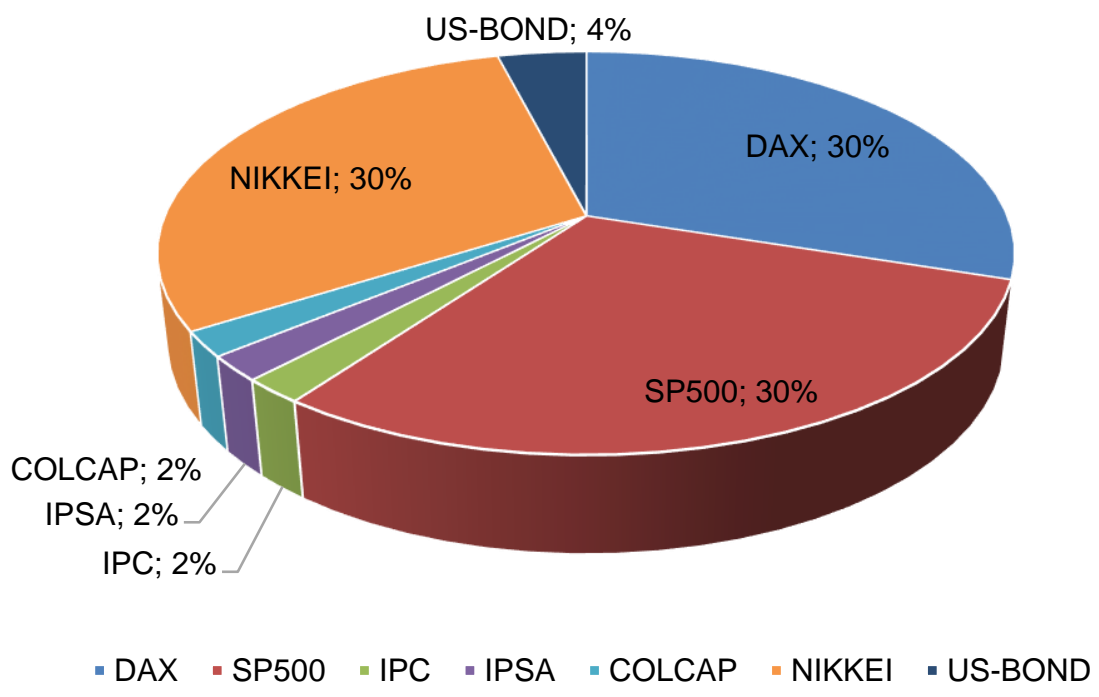

Fig 2: Portafolio óptimo para la cartera seleccionada de índices bursátiles 
El resultado final de la inversión en cada etapa del proyecto se calcula aplicando la ecuación 1 para los ahorros acumulados al final de la etapa anterior más el resultado del ahorro mensual durante el periodo respectivo utilizando la ecuación 2. La experiencia del trabajo semestral ha arrojado que aproximadamente un $70 \%$ de los estudiantes logran cumplir su sueño, el cual generalmente es la cuota inicial de una vivienda, un viaje de vacaciones o reunir el dinero para un emprendimiento personal. El 30\% restante que no cumple su sueño generalmente ha tenido mayores aspiraciones como realizar un posgrado en el exterior o asegurar la educación de uno de sus hijos; se resalta en las conclusiones que la causa no es el sueño por sí mismo, si no la insuficiente cantidad ahorrada, el plazo establecido o que la suerte no acompañó la inversión en bolsa en algunos de los casos.

\section{DISCUSIÓN}

Se toma el ejemplo de uno de los proyectos desarrollados durante el año 2017, en el cual la meta personal era reunir el capital necesario para realizar un posgrado en una universidad pública de España. El plazo es de cinco años y tiene un valor futuro estimado entre 48 y 53 millones de pesos colombianos dependiendo de las consideraciones futuras de inflación y la tasa de cambio. El estudiante decide empezar ahorrando un $20 \%$ de su salario y los resultados por etapa de inversión se muestran en la Tabla 1.

Tabla 1: Resultados por etapa para uno de los trabajos semestrales guiados (en pesos colombianos)

\begin{tabular}{|c|cc|c|c|c|}
\hline & \multicolumn{2}{|c|}{$\begin{array}{c}\text { Ahorro } \\
\text { Etapa }\end{array}$} & $\begin{array}{c}\text { Tipo de } \\
\text { Inversión }\end{array}$ & $\begin{array}{c}\text { Rentabilidad } \\
\text { Periodo }\end{array}$ & $\begin{array}{c}\text { Ahorro } \\
\text { Acumulado }\end{array}$ \\
\hline 1 & $\$$ & 400.000 & Renta fija & $7,80 \%$ & $\$ 4.969 .273$ \\
2 & $\$$ & 416.000 & SP500 & $5,00 \%$ & $\$ 10.323 .129$ \\
3 & $\$$ & 432.640 & SP500 & $30,00 \%$ & $\$ 19.291 .826$ \\
4 & $\$$ & 449.946 & Portafolio & $14,82 \%$ & $\$ 27.907 .564$ \\
5 & $\$$ & 467.943 & Portafolio & $14,82 \%$ & $\$ 38.030 .327$ \\
\hline
\end{tabular}

Este caso estuvo dentro del $30 \%$ de los que no alcanzaron el sueño en el plazo establecido, pero se ilustra para mostrar que lo más importante del trabajo es la reflexión final del estudiante. La primera reflexión es que a pesar de que las rentabilidades obtenidas en las inversiones fueron buenas, sólo logró reunir cerca del $75 \%$ del capital requerido. Sin embargo, el estudiante concluye que le quedan dos alternativas: i) aplazar la meta del sueño por un año más, tiempo en el cual puede llegar a reunir el dinero para su sueño si invierte en un portafolio equivalente al utilizado en las etapas 4 y 5, ii) si insiste en cumplir su meta en cinco años, debe esforzarse más incrementando su ahorro a un $25 \%$ de su salario.

La eficacia del trabajo semestral guiado se midió con una encuesta de 25 preguntas practicada al comienzo y al final del curso. A partir del criterio mostrado en la metodología, esta encuesta mide la percepción de los estudiantes sobre la importancia de la educación financiera, el ahorro, el manejo de las deudas, la rentabilidad y el riesgo; determinando de esta forma que tanta probabilidad tienen los estudiantes de lograr la seguridad financiera. Los resultados son notables pues la encuesta al comienzo del curso arroja que, en promedio, sólo un $25 \%$ de los estudiantes tiene alta probabilidad de lograr la seguridad financiera y al final del curso ese porcentaje se incrementa al $76 \%$ de los estudiantes.

\section{CONCLUSIONES}

Las finanzas personales desempeñan un papel fundamental en el logro del bienestar de los individuos y los hogares, permiten cumplir metas de corto, mediano y largo plazo y contribuyen a disminuir la inequidad a nivel mundial. De ahí que su enseñanza debe partir de la escuela y consolidarse en la universidad, mediante la aplicación de cada uno de los principios básicos de la ingeniería económica.

En este artículo se presentó el análisis de la relación entre rentabilidad y riesgo y la forma de aplicar estos conceptos en un curso de ingeniería económica o matemáticas financieras mediante un trabajo semestral guiado. Los resultados obtenidos permiten que el estudiante tome conciencia de no confiar en las alternativas de inversión con rentabilidades extraordinarias pero que a la vez se pueden asumir riesgos acordes con el grado de aversión al riesgo de cada individuo, su edad y el plazo establecido para la meta.

Los sueños personales se pueden lograr a través de una adecuada planificación financiera. Los trabajos desarrollados por los estudiantes con metas que van desde un viaje soñado por Europa o estudiar un posgrado hasta mejorar los ingresos en la jubilación se lograron en su mayoría gracias a un ahorro adecuado y una estrategia de inversión acorde con la meta específica y el riesgo asumido. 


\section{ANEXO}

En la tabla 2 se muestra el instrumento utilizado para determinar la probabilidad de lograr la seguridad financiera en estudiantes universitarios.

Tabla 2: Instrumento para diagnosticar la seguridad financiera (Adaptado de Samper et al., 2010; Nachtigall, 2016)

\begin{tabular}{|c|c|c|}
\hline \# & Responder verdadero $(V)$ o falso $(F)$ & V \\
\hline 1 & ¿Ahorro una parte de mis ingresos todos los meses? (no importa que estos ingresos sean pequeños) & \\
\hline 2 & ¿Generalmente comparo precios antes de realizar una compra? & \\
\hline 3 & $\begin{array}{l}\text { ¿Suelo aprovechar los cupones de descuento y otras ofertas que permitan generar ahorro en mis } \\
\text { compras? }\end{array}$ & \\
\hline 4 & $\begin{array}{l}\text { ¿Hago una previsión mensual de los gastos que se generan de forma periódica pero que se pueden } \\
\text { predecir (pasajes, alimentos, regalos, etc.)? }\end{array}$ & \\
\hline 5 & ¿Evito gastar dinero en lo que más me gusta sin medir las consecuencias? & \\
\hline 6 & $\begin{array}{l}\text { ¿Tengo una fuente de ingreso adicional, además de la mesada que recibo de mis padres o } \\
\text { patrocinadores? }\end{array}$ & \\
\hline 7 & ¿Gasto menos de lo que recibo? & \\
\hline 8 & ¿Alguna vez he leído un libro de cómo generar riqueza o he leído la bibliografía de alguien rico? & \\
\hline 9 & $\begin{array}{l}\text { ¿Busco consejos (de otras personas, periódicos, web, etc.) que me guíen en el campo de las finanzas } \\
\text { personales y que me ayuden a elevar mi inteligencia financiera? }\end{array}$ & \\
\hline 10 & ¿Tengo un presupuesto mensual de gastos que verifico y actualizo por lo menos una vez al mes? & \\
\hline 11 & ¿Evito al máximo tener que pedir prestado dinero a familiares y amigos? & \\
\hline 12 & ¿Pago a tiempo mis compromisos como deudas o facturas? & \\
\hline 13 & $\begin{array}{l}\text { ¿Analizo previamente cualquier decisión de compra evitando dejarme guiar por la moda o la } \\
\text { tecnología? }\end{array}$ & \\
\hline 14 & $\begin{array}{l}\text { ¿Soy de los que piensa que los juegos de azar como el chance, el bingo, las loterías, etc., son formas } \\
\text { inútiles de buscar la riqueza, por lo tanto, no gasto dinero en ellos? }\end{array}$ & \\
\hline 15 & $\begin{array}{l}\text { ¿Una vez empiece a trabajar como profesional, mi primera inversión será un activo productivo en lugar } \\
\text { de un vehículo particular? }\end{array}$ & \\
\hline 16 & $\begin{array}{l}\text { ¿En el momento de adquirir un crédito, prefiero pagarlo en el menor tiempo posible sin importar que las } \\
\text { cuotas mensuales sean más altas? }\end{array}$ & \\
\hline 17 & $\begin{array}{l}\text { ¿Soy de los que piensa que mis problemas financieros son propios y no tiendo a echar la culpa a otros o } \\
\text { a la situación económica del país? }\end{array}$ & \\
\hline 18 & ¿Suelo aprovechar mi tiempo libre para buscar otras alternativas de mejorar mis ingresos? & \\
\hline 19 & ¿Suelo relacionarme con personas integras, honestas y responsables en el manejo del dinero? & \\
\hline 20 & $\begin{array}{l}\text { ¿Me parece normal y no me causa envidia ni me molesta ver que otras personas tienen mucho dinero y } \\
\text { lo disfrutan plenamente? }\end{array}$ & \\
\hline 21 & ¿Confío en mi capacidad para concretar las metas financieras que me propongo y crecer en la vida? & \\
\hline 22 & $\begin{array}{l}\text { ¿Las personas que me conocen consideran que soy un persona persistente, que sabe batallar por lo que } \\
\text { quiere en la vida? }\end{array}$ & \\
\hline 23 & ¿Destino una parte de mi tiempo a capacitarme financieramente? & \\
\hline 24 & $\begin{array}{l}\text { ¿Me gusta y acostumbro investigar sobre nuevas maneras de generar dinero de manera equilibrada y } \\
\text { próspera? }\end{array}$ & \\
\hline 25 & ¿Controlo mis gastos diarios de manera que me siento tranquilo(a) y organizado(a) financieramente? & \\
\hline
\end{tabular}

\section{AGRADECIMIENTOS}

Los autores agradecen el apoyo financiero recibido de la Universidad de Antioquia por medio del proyecto "Sostenibilidad 2018-2019". 


\section{REFERENCIAS}

Altfest, L. J., Personal Financial Planning, 2ª Ed., McGraw-Hill, New York, NY, USA (2017)

Aren, S. y A. N. Zengin, Influence of Financial Literacy and Risk Perception on Choice of Investment, Procedia - Social and Behavioral Sciences, 235, 656-663 (2016)

Bellofato, A., C. D'Hondt y R. De Winne, Subjective Financial Literacy and Retail Investor's Behavior, Journal of Banking and Finance, 92, 168-181 (2018)

Chan, A., Educación Financiera para Padres e Hijos, 1aㅡ Ed., Plataforma Editorial, Barcelona, España (2016)

Chang, B., T. L. Webb, Y. Benn y P. P. Reynolds, Monitoring Personal Finances: Evidence that Goal Progress and Regulatory Focus Influence when People Check their Balance, Journal of Economic Psychology, 62, 33-49 (2017)

Damodaran, A., Annual Returns on Stock, T.Bonds and T.Bills: 1928 - current, 12 de febrero (2018)

Ergun, K., Financial Literacy among University Students: A Study in Eight European Countries, International Journal of Consumer Studies, 42, 2-15 (2018)

Garay, G., Índice de Alfabetismo Financiero, la Cultura y la Educación Financiera, Perspectivas, 19 (37), 23-40 (2016)

Guthrie, C. P. y C. M. Nicholls, The Personal Budget Project: A Practical Introduction to Financial Literacy, Journal of Accounting Education, 33, 138-163 (2015)

He, X. Z. y L. Shi, Index Portfolio and Welfare Analysis under Heterogeneous Beliefs, Journal of Banking and Finance, 75 , 64-79 (2017)

Hsiao, Y. J. y W. C. Tsai, Financial Literacy and Participation in the Derivatives Markets, Journal of Banking and Finance, 88, 15-29 (2018)

Kiyosaki, R., Awaken your Child's Financial Genius, 1를., Plata Publishing, Scottsdale, AZ, Estados Unidos (2012)

Marcowitz, H., Portfolio Selection, Journal of Finance, 7, (1952)

Nachtigall, P., Inteligencia Emocional Financiera, 1aㅡ. Ed., Diana, Bogotá, Colombia (2016)

Palacios, J., Finanzas Personales: Cuatro Principios para Invertir Bien, 1aㅡ Ed., 17-31. LID, Madrid, España (2012)

Samper, M. J., C. Pinto, J. Arenas y M. Roca, Mis Finanzas Personales, 1aㅡ Ed., 37-71. Aguilar, Bogotá, Colombia (2010)

Stolper, O. y A. Walter, Financial Literacy, Financial Advice and Financial Behavior, Journal of Business Economics, 87 (5), 581-643 (2017)

Torres, J., D., D. Acevedo y P. M. Montero, Proyectos de Aula Semestrales como Estrategia Pedagógica para la Formación en Ingeniería, Formación Universitaria, 9 (3), 23-30 (2016)

Valderrrama, A. y J. Valderrama, Trabajo Semestral Guiado: un Buen Complemento al Estudio y Aprendizaje en Cursos de Ciencia y de Ingeniería, Formación Universitaria, 7 (3), 3-10 (2014)

Vargas, M. y B. L. Avendaño, Diseño y Análisis de Psicométrico de un Instrumento que Evalúa Competencias Básicas en Economía y Finanzas: una Contribución a la Educación para el Consumo, Universitas Psychologica, 13 (4), 15-30 (2014)

Villada, F., J. M. López-Lezama y N. Muñoz-Galeano, El Papel de la Educación Financiera en la Formación de Profesionales de la Ingeniería, Formación Universitaria, 10 (2), 13-22 (2017)

Zicari, A. P., Finanzas Personales y Ciclo de Vida: un Desafío Actual, Invenio, 11 (20), 63-71 (2008) 
